\title{
A Note on Correlations between Eigenvalues of a Random Matrix
}

\author{
M. L. MeHTA \\ Service de Physique Théorique Centre d'Etudes Nucléaires de Saclay, Gif-sur-Yvette
}

Received October 1, 1970

\begin{abstract}
Dyson's method is adopted here for the so called Gaussian ensembles. Incidently this confirms the long cherished belief that the statistical properties of a small number of eigenvalues is the same for the two kinds of ensembles, the circular and the Gaussian ones.
\end{abstract}

In studies of the statistical behaviour of the eigenvalues of random matrices, some authors have used as a basis the Gaussian matrix ensembles [1] while others have used the circular ensembles [2]. The Gaussian ensembles have a clearer physical motivation, while the circular ensembles are mathematically simpler. The choice of ensemble has been a matter of personal taste, and it has never been made clear how far the predictions of the theory might depend on the ensemble which is chosen. We here demonstrate that the predictions of Gaussian and circular ensembles in fact become identical in the limit as the order $N$ of the matrices tends to infinity. More precisely, we prove that for any fixed $n$ the joint probability density function of $n$ eigenvalues in the Gaussian ensemble of order $N$ tends to the same limit when $N \rightarrow \infty$ as the corresponding density function in the circular ensemble. This means that in an infinitely long eigenvalue sequence all the statistical properties are independent of the choice of ensemble.

Recently Dyson derived explicit analytical expressions for the joint probability density functions of $n$ eigenvalues belonging to a random matrix taken from the circular ensembles [3]. We indicate below the necessary changes in his equations to give explicit expressions for the same correlation functions the random matrix being taken this time from the corresponding Gaussian ensembles. These changes do not alter in any way his arguments and we write therefore only the equations which are changed and which replace those in Dyson's paper with the same numbering. This note is supposed to be read along with Dyson's original.

$$
\begin{gathered}
x_{j}=\frac{\pi}{(2 N)^{\frac{1}{2}}} \frac{1}{D} E_{j}, \\
Q_{N \beta}\left(x_{1}, \ldots, x_{N}\right)=C_{N \beta} \exp \left(-\frac{1}{2} \beta \sum_{1}^{N} x_{j}^{2}\right) \prod_{j<k}\left|x_{j}-x_{k}\right|^{\beta} .
\end{gathered}
$$


Everywhere in the text $\theta_{j}$ is to be replaced by $x_{j}$.

$$
C_{N \beta}=(2 \pi)^{\frac{1}{2} N} \beta^{-\frac{1}{2} N-\frac{1}{4} \beta N(N-1)}\left\{\Gamma\left(1+\frac{1}{2} \beta\right)\right\}^{-N} \prod_{j=1}^{N} \Gamma\left(1+\frac{1}{2} \beta j\right) \text {. }
$$

The limits of integration in Eq. (1.8) are $(-\infty, \infty)$.

$$
R_{N 0 \beta}=1 \text {, }
$$

$R_{N 11}\left(x_{1}\right)=\sum_{j=0}^{N-1} \varphi_{j}^{2}\left(x_{1}\right)+\left(\frac{1}{2} N\right)^{\frac{1}{2}} \varphi_{N-1}(x)\left(\int_{-\infty}^{x_{1}}-\int_{x_{1}}^{\infty}\right) \varphi_{N}(t) d t$,

$R_{N 12}\left(x_{1}\right)=\sum_{j=0}^{N-1} \varphi_{j}^{2}\left(x_{1}\right)$,

$R_{N 14}\left(x_{1}\right)=\frac{1}{\sqrt{2}} \sum_{j=0}^{2 N-1} \varphi_{j}^{2}\left(x_{1} \sqrt{2}\right)+\frac{1}{\sqrt{2}} N^{\frac{1}{2}} \varphi_{2 N}\left(x_{1} \sqrt{2}\right) \int_{-\infty}^{x_{1} \sqrt{2}} \varphi_{2 N-1}(t) d t$, where

$$
\varphi_{j}(x)=\left(\pi^{\frac{1}{2}} 2^{j} j !\right)^{-\frac{1}{2}} e^{x^{2} / 2}\left(\frac{d}{d x}\right)^{j} e^{-x^{2}}
$$

are the normalized harmonic oscillator wave functions.

$$
P_{N \beta}\left(E_{1}, \ldots, E_{n}\right)=\operatorname{Lim}_{N \rightarrow \infty}\left(\frac{\pi}{D(2 N)^{\frac{1}{2}}}\right)^{n} R_{N n \beta}\left(x_{1}, \ldots, x_{n}\right) .
$$

The text following Eq. (1.10) and the whole of Section II remains unchanged. The Eq. (3.1) is replaced by

$$
S_{N}(x, y)=\sum_{j=0}^{N-1} \varphi_{j}(x) \varphi_{j}(y)+\left(\frac{1}{2} N\right)^{\frac{1}{2}} \varphi_{N-1}(x) \int_{-\infty}^{\infty} \varepsilon(y, t) \varphi_{N}(t) d t,
$$

where

$$
\varepsilon(x, y)=\frac{1}{2} \operatorname{Sign}(x-y)=\left\{\begin{aligned}
\frac{1}{2}, & x>y, \\
0, & x=y, \\
-\frac{1}{2}, & x<y .
\end{aligned}\right.
$$

In Section III we make the following changes

$$
\begin{aligned}
D S_{N}(x, y) & =-\frac{d}{d y} S_{N}(x, y)=-D S_{N}(y, x), \\
I S_{N}(x, y) & =\int_{-\infty}^{\infty} \varepsilon(x, t) S_{N}(t, y) d t=-I S_{N}(y, x), \\
J S_{N}(x, y) & =I S_{N}(x, y)-\varepsilon(x, y),
\end{aligned}
$$




$$
\begin{aligned}
& \sigma_{N 1}(x, y)=\left[\begin{array}{ll}
S_{N}(x, y)+\alpha(x) & D S_{N}(x, y) \\
J S_{N}(x, y) & S_{N}(y, x)+\alpha(y)
\end{array}\right], \\
& \sigma_{N 4}(x, y)=\frac{1}{\sqrt{2}}\left[\begin{array}{cc}
S_{2 N+1}(x \sqrt{2}, y \sqrt{2}) & D S_{2 N+1}(x \sqrt{2}, y \sqrt{2}) \\
I S_{2 N+1}(x \sqrt{2}, y \sqrt{2}) & S_{2 N+1}(y \sqrt{2}, x \sqrt{2})
\end{array}\right], \\
& \sigma_{N 2}(x, y)=\sum_{j=0}^{N-1} \varphi_{j}(x) \varphi_{j}(y),
\end{aligned}
$$

where

$$
\alpha(x)=\varphi_{2 m}(x) / \int_{-\infty}^{\infty} \varphi_{2 m}(t) d t, \quad \text { for } \quad N=2 m+1 \quad \text { odd }
$$

and

$$
\alpha(x)=0, \text { for } N=2 m \text { even . }
$$

The interchange of $(x, y)$ in the lower right hand corner of Eqs. (3.16) and (3.17) is to be noticed. We study the quaternion determinants

$$
U_{N n \beta}\left(x_{1}, \ldots, x_{n}\right)=Q \operatorname{Det}\left[\sigma_{N}\left(x_{j}, x_{k}\right)\right]_{j, k=1, \ldots, n},
$$

which are functions of the $n$ variables $x_{1}, \ldots, x_{n}$.

Theorem 3 takes the form

For $\beta=1,2,4$,

$$
U_{N N \beta}\left(x_{1}, \ldots, x_{N}\right)=Q_{N \beta}\left(x_{1}, \ldots, x_{N}\right),
$$

with $Q_{N \beta}$ given by Eq. (1.2).

In the proof of Theorem 3 we make the following changes. Case $\beta=1, N=2 m$, even.

$$
\begin{aligned}
& P=\left[\begin{array}{lll}
\varphi_{2 i}\left(x_{j}\right) & \varphi_{2 i}^{\prime}\left(x_{j}\right) & 0 \\
\int_{0}^{x_{j}} \varphi_{2 i}(t) d t & \varphi_{2 i}\left(x_{j}\right) & 0
\end{array}\right]\left[\begin{array}{cc}
\varphi_{2 i}\left(x_{k}\right) & -\varphi_{2 i}^{\prime}\left(x_{k}\right) \\
-\int_{0}^{x_{k}} \varphi_{2 i}(t) d t & \varphi_{2 i}\left(x_{k}\right) \\
0 & 0
\end{array}\right] \\
& =\left[\begin{array}{rr}
S_{N} & D S_{N} \\
I S_{N} & S_{N}^{T}
\end{array}\right] \equiv\left[\begin{array}{rr}
S_{N}\left(x_{j}, x_{k}\right) & D S_{N}\left(x_{j}, x_{k}\right) \\
I S_{N}\left(x_{j}, x_{k}\right) & S_{N}\left(x_{k}, x_{j}\right)
\end{array}\right]
\end{aligned}
$$

has rank $N$, and

so that

$$
\left[D S_{N}\left(x_{j}, x_{k}\right)\right]=\left[\varphi_{2 i}\left(x_{j}\right) \quad \varphi_{2 i}^{\prime}\left(x_{j}\right)\right]\left[\begin{array}{r}
-\varphi_{2 i}^{\prime}\left(x_{k}\right) \\
\varphi_{2 i}\left(x_{k}\right)
\end{array}\right]
$$

$$
\operatorname{det}\left[D S_{N}\left(x_{j}, x_{k}\right)\right]=\left\{\operatorname{det}\left[\varphi_{2 i}\left(x_{j}\right) \quad \varphi_{2 i}^{\prime}\left(x_{j}\right)\right]\right\}^{2} .
$$

The recurrence relation

$$
\sqrt{2 i+1} \varphi_{2 i+1}(x)=\sqrt{2 i} \varphi_{2 i-1}-\sqrt{2} \varphi_{2 i}^{\prime}(x)
$$


shows that in a determinant containing the rows $\varphi_{2 i+1}(x), i=0,1, \ldots, j-1$ one can replace the row $\varphi_{2 j+1}(x)$ by the row $-\left(\frac{2}{2 j+1}\right)^{\frac{1}{2}} \varphi_{2 j}^{\prime}(x)$, and vice-versa. Thus apart from a constant the rows $\varphi_{2 j+1}(x), j=0,1,2, \ldots$ are equivalent to the rows $\varphi_{2 j}^{\prime}(x), j=0,1,2, \ldots$ when they occur in a determinant.

Thus

$$
\operatorname{det}\left[D S_{N}\left(x_{j}, x_{k}\right)\right]=\text { const. }\left\{\operatorname{det}\left[\varphi_{j}\left(x_{k}\right)\right]\right\}^{2}=\text { const. }\left\{Q_{N 1}\left(x_{1}, \ldots, x_{N}\right)\right\}^{2} .
$$

Case $\beta=1, N=2 m+1$ odd.

$$
\begin{aligned}
& P_{\delta}=\left[\begin{array}{llll}
\varphi_{2 i+1}\left(x_{j}\right) & \varphi_{2 i+1}^{\prime}\left(x_{j}\right) & \sqrt{\delta} \varphi_{2 m}\left(x_{j}\right) & 0 \\
\int_{-\infty}^{x_{j}} \varphi_{2 i+1}(t) d t & \varphi_{2 i+1}\left(x_{j}\right) & -\left(\sqrt{\delta} \int_{-\infty}^{\infty} \varphi_{2 m}(t) d t\right)^{-1} & 0
\end{array}\right] \\
& \times\left[\begin{array}{cc}
\varphi_{2 i+1}\left(x_{k}\right) & -\varphi_{2 i+1}^{\prime}\left(x_{k}\right) \\
-\int_{-\infty}^{x_{k}} \varphi_{2 i+1}(t) d t & \varphi_{2 i+1}\left(x_{k}\right) \\
\left(\sqrt{\delta} \int_{-\infty}^{\infty} \varphi_{2 m}(t) d t\right)^{-1} & -\sqrt{\delta} \varphi_{2 m}\left(x_{k}\right) \\
0 & 0
\end{array}\right] \\
& =\left[\begin{array}{cc}
S_{N}\left(x_{j}, x_{k}\right)+\alpha\left(x_{j}\right) & D S_{N}\left(x_{j}, x_{k}\right)+\delta \varphi_{2 m}\left(x_{j}\right) \varphi_{2 m}\left(x_{k}\right) \\
I S_{N}\left(x_{j}, x_{k}\right)+\left(\sqrt{\delta} \int_{-\infty}^{\infty} \varphi_{2 m}(t) d t\right)^{-2} & S_{N}\left(x_{k}, x_{j}\right)+\alpha\left(x_{k}\right)
\end{array}\right] \text {, } \\
& A_{\delta}=\left[\begin{array}{ll}
S_{N}\left(x_{j}, x_{k}\right)+\alpha\left(x_{j}\right) & D S_{N}\left(x_{j}, x_{k}\right)+\delta \varphi_{2 m}\left(x_{j}\right) \varphi_{2 m}\left(x_{k}\right) \\
J S_{N}\left(x_{j}, x_{k}\right) & S_{N}\left(x_{k}, x_{j}\right)+\alpha\left(x_{k}\right)
\end{array}\right] .
\end{aligned}
$$

Therefore by the argument following Eq. (3.32) we have:

$$
\begin{aligned}
\operatorname{det} A_{\delta}= & \operatorname{det}\left[\varepsilon\left(x_{j}, x_{k}\right)-\frac{1}{\delta}\left(\int_{-\infty}^{\infty} \varphi_{2 m}(t) d t\right)^{-2}\right] \\
& \times \operatorname{det}\left[D\left(x_{j}, x_{k}\right)+\delta \varphi_{2 m}\left(x_{j}\right) \varphi_{2 m}\left(x_{k}\right)\right] .
\end{aligned}
$$

The first factor on the right of Eq. (3.33) is given by (3.38), while the second factor is:

$$
\begin{gathered}
\left\{\begin{array}{c}
\operatorname{det}\left[\varphi_{2 i+1}\left(x_{j}\right) \quad \varphi_{2 i+1}^{\prime}\left(x_{j}\right) \quad \sqrt{\delta} \varphi_{2 m}\left(x_{j}\right)\right]_{\substack{i=0,1, \ldots, m-1 \\
j=1,2, \ldots, 2 m+1}}
\end{array}\right\}^{2} \\
=\delta\left\{\operatorname{det}\left[\varphi_{i-1}\left(x_{j}\right)\right]_{i, j=1, \ldots, N}\right\}^{2} \times \text { (const.). }
\end{gathered}
$$


In obtaining the last step we have used the fact that $\varphi_{2 i+1}^{\prime}(x)$ is a linear combination of $\varphi_{2 i}(x)$ and $\varphi_{2 i+2}(x)$; the presence of $\varphi_{2 m}(x)$ allowing us to eliminate then successively the $\varphi_{2 i+2}(x)$.

Case $\beta=4$.

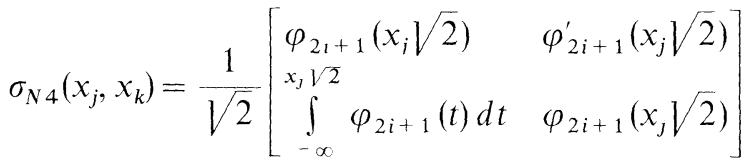

$$
\begin{aligned}
& \times\left[\begin{array}{cc}
\varphi_{2 i+1}\left(x_{k} \sqrt{2}\right) & -\varphi_{2 i+1}^{\prime}\left(x_{k} \sqrt{2}\right) \\
\int_{-\infty}^{x_{k} \sqrt{2}} \varphi_{2 i+1}(t) d t & \varphi_{2 i+1}\left(x_{k} \sqrt{2}\right)
\end{array}\right] .
\end{aligned}
$$

So that

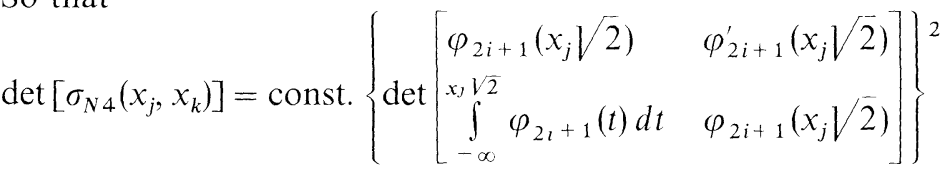

$$
\begin{aligned}
& =\text { const. }\left\{\operatorname{det}\left[\begin{array}{ll}
\varphi_{2 i+1}\left(x_{j} \sqrt{2}\right) & \varphi_{2 i+1}^{\prime}\left(x_{j} \sqrt{2}\right) \\
\varphi_{2 i}\left(x_{j} \sqrt{2}\right) & \varphi_{2 i}^{\prime}\left(x_{j} \sqrt{2}\right)
\end{array}\right]\right\}^{2} \\
& =\text { const. }\left\{\operatorname{det}\left[\varphi_{j}\left(x_{k} \sqrt{2}\right) \quad \varphi_{j}^{\prime}\left(x_{k} \sqrt{2}\right)\right]\right\}^{2} \\
& \text { = const. }\left\{Q_{N 4}\left(x_{1}, \ldots, x_{N}\right)\right\}^{2} \text {. }
\end{aligned}
$$

In the second step above we have used the fact that in a determinant the rows $\varphi_{2 i+1}(x)$ are equivalent to the rows $\varphi_{2 i}^{\prime}(x)$, apart from a constant.

In Section IV we write Eq. (4.4) as

$$
\left(f_{1} * f_{2}\right)(x, z)=\int_{-\infty}^{\infty} f_{1}(x, y) f_{2}(y, z) d y .
$$

Then Eqs. (4.5) to (4.10) are valid, while Eq. (4.11) is replaced by:

$$
\sigma_{N 1} * \sigma_{N 1}=\sigma_{N 1}+E,
$$

where $E(x, y)$ is an anti-symmetric matrix. The terms cancel each other exactly on summing over the $(n-1)$ ! cyclic permutations of $\left(x_{1}, \ldots, x_{n}\right)$ in Eq. (4.2) which should evidently be read as

$$
V_{N n \beta}\left(x_{1}, \ldots, x_{N}\right)=\sum_{P}\left[\sigma_{N \beta}\left(x_{1}, x_{2}\right) \ldots \sigma_{N \beta}\left(x_{n}, x_{1}\right)\right] .
$$

The rest of Section IV is unchanged.

Note. Keeping $x_{j} N^{\frac{1}{2}}$ and $\theta_{j} N$ fixed at finite values for $j=1, \ldots, n$ and taking the limit $N \rightarrow \infty$, all the correlation functions $P_{n \beta}$ are identical for the Gaussian and the circular ensembles [4]. Thus the two ensembles give the same statistical properties. 
Acknowledgements. I am thankful to my collegues B. Antoine and J. F. Renardy for having the patience to listen to me and make critical remarks.

\section{References}

1. Wigner, E. P.: Ann. Math. 62, 548 (1955); 65, 203 (1957); SIAM Review 9, 1 (1967).

Mehta, M. L.: Random matrices and the statistical theory of energy levels. New York: Academic Press 1967.

Porter, C. E.: First chapter of Statistical Theories of Spectra: Fluctuations. New York: Academic Press 1965.

Gaudin, M.: Nucl. Phys. 25, 447 (1961).

2. Dyson, F. J.: J. Math. Phys. 3, 140 (1962); 3, 157 (1962); 3, 166 (1962); 3, 1191 (1962); 3, 1199 (1962).

-- Mehta, M. L.: J. Math. Phys. 4, 701 (1963); 4, 713 (1963).

3. - Correlations between eigenvalues of a random matrix. Commun. math. Phys. 19, 235- 250 (1970).

4. Mehta, M. L.: Reference 1, Appendix A. 9.

M. L. Mehta

Service de Physique Théorique

Centre d'Etudes Nucléaires de Saclay

B.P. No. 2

Gif-sur-Yvette, France 